\title{
Fast Automated Segmentation and Reproducible Volumetry of Pulmonary Metastases in CT-Scans for Therapy Monitoring
}

\author{
Jan-Martin Kuhnigk ${ }^{1}$, Volker Dicken ${ }^{1}$, Lars Bornemann ${ }^{1}$, Dag Wormanns ${ }^{2}$, \\ Stefan Krass ${ }^{1}$, and Heinz-Otto Peitgen ${ }^{1}$ \\ ${ }^{1}$ MeVis, 28359 Bremen, Germany, kuhnigk@mevis . de \\ 2 Institute for Clinical Radiology, University of Muenster, Germany
}

\begin{abstract}
The assessment of metastatic growth under chemotherapy belongs to the daily radiological routine and is currently performed by manual measurements of largest nodule diameters. As in lung cancer screening where $3 \mathrm{~d}$ volumetry methods have already been developed by other groups, computer assistance would be beneficial to improve speed and reliability of growth assessment. We propose a new morphology and model based approach for the fast and reproducible volumetry of pulmonary nodules that was explicitly developed to be applicable to lung metastases which are frequently large, not necessarily spherical, and often complexly attached to vasculature and chest wall. A database of over 700 nodules from more than 50 patient $\mathrm{CT}$ scans from various scanners was used to test the algorithm during development. An in vivo reproducibility study was conducted concerning the volumetric analysis of 105 metastases from 8 patients that were subjected to a low dose CT scan twice within several minutes. Low median volume deviations in inter-observer $(0.1 \%)$ and inter-scan ( $4.7 \%)$ tests and a negligible average computation time of 0.3 seconds were measured. The experiments revealed that clinically significant volume change can be detected reliably by the method.
\end{abstract}

\section{Introduction}

Since the entire cardiac output flows through the lungs, the risk of hematogenous metastases is very high. Apart from primary lung cancer, the most common tumors involving the lung parenchyma are breast cancer, gastrointestinal tumors, kidney cancer, melanoma, sarcomas, lymphomas and leukemias, and germ cell tumors. Because of the systemic character of the disease, chemotherapy is the standard treatment for lung metastases. To assess the effect of chemotherapy, a follow-up examination is performed typically 3-6 months after the start of the treatment. Tumor growth is the standard decision parameter on whether the current therapy is successful and to be continued, or not, in which case the therapy has to be modified. A similar question arises in the context of lung cancer screening, where nodule growth is used as an indicator for malignancy.

Being a 3-dimensional process, volume growth assessment is a challenging task in $2 \mathrm{~d}$ CT slices. Current standard criteria (RECIST ${ }^{1}$ ) require the radiologist to locate the

\footnotetext{
${ }^{1}$ Response Evaluation Criteria in Solid Tumors, see http://ctep.cancer.gov/guidelines/recist.html for further information.
} 

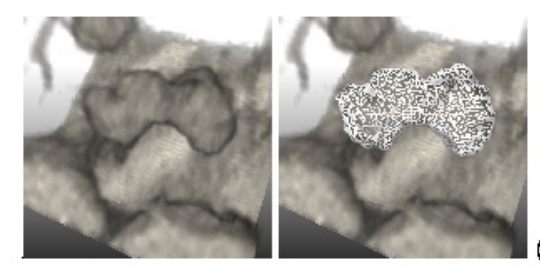

(a)
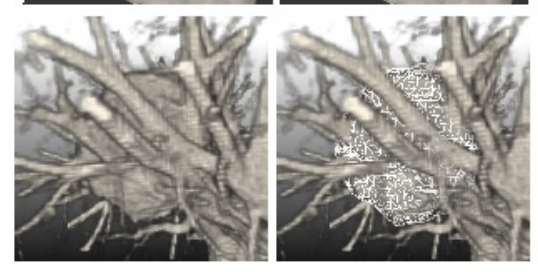
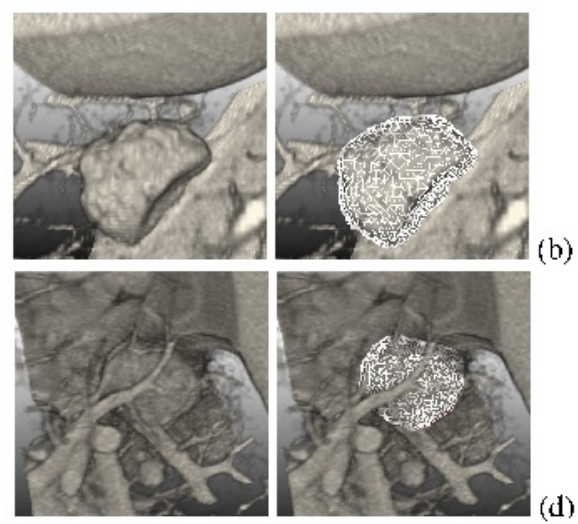

(d)

Fig. 1. 3d visualization of segmentation results for large metastases with irregular shape $(a, b)$, extensive contact to the chest wall (a, d), a lobar fissure (b) and complex vasculature (c, d).

five largest tumors in the lung and find the axial slice where the tumor appears largest, in order to manually measure the greatest nodule diameter. Even if measuring errors are neglected, the $2 \mathrm{~d}$ criteria are reliable only for spherical nodules and do generally fail for irregularly shaped nodules. Additionally, manually finding the correct slice and measuring $2 \mathrm{~d}$ diameters for each of possibly many nodules not only leads to reproducibility issues but is also time-consuming. In order to assess nodule growth quickly and reliably, computer assistance for reproducible $3 \mathrm{~d}$ volumetry is desirable.

The need for convenient and reliable growth estimation in the context of lung cancer screening has resulted in the development and evaluation of algorithms for volume assessment of small lesions. Examples of well evaluated algorithms specifically designed for the $3 \mathrm{~d}$ segmentation of small pulmonary nodules can be found in [1] and [2]. For small nodules that consist mostly of partial volume in CT scans, the step from segmentation to volumetry is especially challenging. Ko et al. [3] compared different volume measurement methods in a phantom study and found that methods with an explicit model for partial volumes (partial-volume methods, PVM) perform significantly superior to fixed-threshold methods.

In contrast to the asymptomatic screening population the segmentation approaches mentioned above were primarily designed for, the population of patients undergoing chemotherapy typically suffers from advanced inoperable cancer. Metastatic tumors occur at all stages, so that algorithms have to deal with the full range of appearances, from small spherical nodules $(\varnothing<10 \mathrm{~mm}$ ) consisting mostly of partial volume voxels, to large nodules $(\varnothing>40 \mathrm{~mm})$ of irregular morphology. Due to their size, the latter are more likely to be complexly connected to vasculature and chest wall, structures which are in most cases indistinguishable from the nodule with respect to density values in CT scans. Additionally, performance issues arise, since the volume of interest to be analyzed for a $40 \mathrm{~mm}$ nodule is about $4^{3}=64$ times larger than for a $10 \mathrm{~mm}$ nodule.

Our approach does not include the computer aided detection $(C A D)$ of nodules, but can be combined easily with existing CAD methods, examples of which can be found in [4] and [5]. Furthermore, the decision was made not to incorporate information from 
global lung structure analyses. Whilst robust methods in that area exist ([6], [7]) which could provide helpful information about the nodule location (such as proximity to the chest wall or a lobar fissure), any analysis performed by our method is restricted to the volume of interest. This allows for a simple integration into the workflow of existing clinical workstations.

In the following, the new segmentation procedure is explained in detail. Morphological methods are combined with model based considerations to achieve a robust separation of the nodule from attached structures. To assess the volume of the segmented lesion we developed a method similar to the procedure described by Ko et al. in [3].

\section{Methods}

\subsection{Preliminaries}

The algorithm works on a cubic input volume (VOI, Volume of Interest). The set $V$ denotes the set of all voxels within the input volume. Density values range from -1024 Hounsfield Units (HU) to $3071 \mathrm{HU}$. The algorithm works under the assumption that $V$ contains the complete nodule. The seed point $s$ is required to be located in the nodule.

The elementary image processing methods included in the segmentation procedure, i. e., thresholding, region growing, connected component analysis, convex hull, Euclidean distance transform, are described and discussed in [8].

\subsection{Preprocessing}

An initial segmentation is performed using a region growing algorithm with a fixed lower threshold of $-450 \mathrm{HU}$ starting from the seed point $s$. The result is an initial set of voxels $N_{0}$, the first estimate of the nodule region (Figure $2 \mathrm{~b}$ ).

Subsequently, a connected component analysis (also called region identification) is performed on the complement of $N_{0}$. The largest connected non-segmented area $P$ is extracted. It corresponds to the largest connected area of lung parenchyma in the VOI surrounding the nodule of interest (Figure 2 c). For the segmentation, $P$ is used in two ways: First, for nodules with no chest wall attachment, $P$ is basically the complement of $N_{0}$, except for dark areas (such as necrosis or noise) within the nodule. We define a superset $N_{1}:=V-P$ of $N_{0}$, which is essentially $N_{0}$ with its gaps closed. Second, for nodules with chest wall attachment, $P$ is crucial to the subsequent separation procedure.

\subsection{Separation from the Chest Wall}

As the example in Figure 2 shows, not only adjacent vasculature but also parts of the chest wall can be included in the initial segmentation result $N_{0}$. CT images generally do not show any visible density contrast between the nodule and the attached chest wall (2). Even expert radiologists are usually unable to determine whether a nodule is still separated from the chest wall by the pleura, or if the chest wall was infiltrated. Thus, we decided to focus on reproducibly segmenting the part of the nodule surrounded by lung parenchyma. In order to achieve this goal, the algorithm makes use of the anatomical fact that the lungs are mostly 2 convex, while the surrounding parenchyma, defined by

\footnotetext{
${ }^{2}$ Noteworthy exceptions to this rule are the diaphragm and cardiac region.
} 

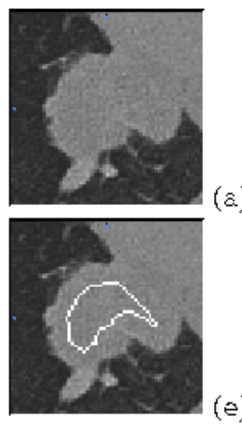
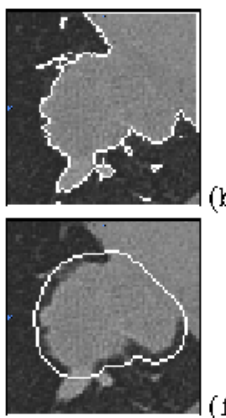
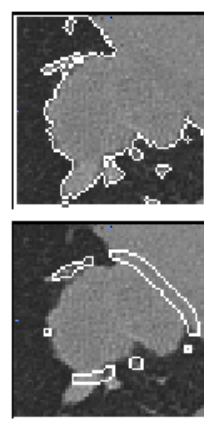
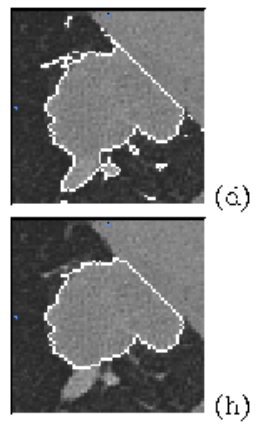

Fig. 2. The essential steps of the segmentation procedure visualized on a single slice. In each of the images (b) to (h), the area enclosed by highlighted boundaries corresponds to a specific set of voxels:

(a) The situation: A pulmonary nodule with contact to both chest wall and vasculature.

(b) $N_{0}:$ Result of the initial $3 \mathrm{~d}$ region growing.

(c) $P \quad$ : Complement of $N_{0}$ (in this case).

(d) $N_{2}$ : Intersection of $N_{0}$ with the convex hull of $P$.

(e) $N_{-}:$Result after the $3 \mathrm{~d}$ erosion procedure.

(f) $N_{+}$: Result of the 3 d dilation (using the secondary distance map $D$ ).

(g) $I_{\varepsilon} \quad$ : Dilated intersection of the boundaries of $N_{+}$(Fig. f) with the initial mask $N_{0}$ (Fig.b).

(h) $N_{\star}:$ Refined segmentation result, obtained by combination of $\mathrm{f}$ and $\mathrm{g}$.

$P$, shows at least one major concavity: the nodule itself. To remove concavities from an object, a convex hull operation can be used. It provides the minimal extension of a set $S$ to a convex set that contains all points of $\mathrm{S}$. The mask convex hull of $P$ is used to mask out the chest wall part from $N_{1}$ and provide an improved estimate of the nodule region $N_{2}:=N_{1} \cap$ convexHull $(P)$. The result can be seen in Figure $2 \mathrm{~d}$.

\subsection{Separation from Attached Vasculature}

Since the pleura separation is only capable of masking out structures that are not part of the lungs, adjacent vessels are still included in $N_{2}$. Since the algorithm aims specifically at the volumetry of large pulmonary metastases, extensive connections to vasculature are to be expected (as in Figures 1 , and $1 \mathrm{~d}$ ).

The density information in CT images does not suffice to allow a density-based separation procedure. However, nodules and vessels differ significantly in morphology. By making use of the fact that the nodule's connection to the vasculature is usually thinner than the nodule itself, a separation of the structures is performed by morphological opening (erosion followed by dilation). Two distance transformations were used to implement the erosion and dilation. A similar procedure was used to label aneurysm voxels in [9]. A primary 3d Euclidean distance transform $E$ is performed to compute the minimum distance from each voxel in $N_{1}$ to the background. World coordinates are used in order to account for voxel anisotropy:

$$
E(v):=\min \left\{\left\|\operatorname{world}(v)-\operatorname{world}\left(v^{\prime}\right)\right\|_{2}: v^{\prime} \notin N_{1}\right\} .
$$




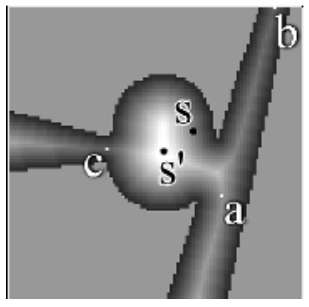

Schematic view of a nodule with vascular connection showing the primary distances in $E$ represented by gray values, the initial seed point $s$, the corrected seed point $s^{\prime}$ as well as the three points $\mathrm{a}, \mathrm{b}, \mathrm{c}$, indicating the minimum vessel radius on any path from $s^{\prime}$ to the VOI boundaries. The greatest distance value of all three points, in this case $E(a)$, provides the lower boundary for the erosion strength, since its application would remove the connections to any of the attached vessels.

Fig. 3. Vascular connectivity model.

The erosion is implemented by applying a threshold to the distance map. The crucial part of the operation is to find an optimal erosion threshold in order to cut off unwanted adjacent structures without clipping significant nodule boundary features. The determination of the threshold will be discussed in detail in the following.

Starting from the given seed point $s$, a local maximum search is performed on the distance map yielding a new seed point $s^{\prime}$ (Figure 3). For the determination of the optimal erosion threshold $t_{\star}$, a model was established (see also Figure 3) which makes the following two assumptions with respect to vascular attachment: (1) Each lung vessel is ultimately originating in the hilum region. Hence, each vessel connected to the nodule is also connected to the VOI boundaries. (2) Each vessel's diameter is monotonic decreasing with the increasing distance to the hilum. Thus, if a vessel ends at the nodule, its diameter is smallest at its entry point into the nodule (point $\mathbf{c}$ in Figure 3). If a vessel only touches a nodule, the smallest diameters along the two possible paths from the touching point to the VOI boundaries are located directly at the boundaries (point $\mathbf{b}$ in Figure 3) and in close proximity to the nodule (point a in Figure 3). Given assumption (2), the latter is bigger than the former. Hence, by using the maximum of all minimal path diameters as a lower bound for the erosion strength parameter, we ensure that vessels are disconnected by the erosion in close proximity to their connection with the nodule. We will refer to this erosion threshold by $t_{\star}$, since it is optimal with respect to the proposed model. The proposed procedure implies the limitation that a nodule cannot be separated from a broadly attached vessel that has a larger radius than the nodule itself. However, these cases appear to be extremely rare 3 . To actually compute $t_{\star}$, a variable threshold region growing was performed on $E$ which determines the highest threshold sufficient to reach the VOI boundaries from $s^{\prime}$. To be less susceptible to noise and discretization artifacts, the actual erosion threshold $t_{-}$is chosen slightly greater than $t_{\star}$.

To perform the second step of the opening, the dilation, a secondary distance map $D$ is generated, mapping each voxel to its distance to the eroded nodule mask $N_{-}$:

$$
D(v):=\min \left\{\left\|\operatorname{world}(v)-\operatorname{world}\left(v^{\prime}\right)\right\|_{2}: v^{\prime} \in N_{-}\right\} .
$$

\subsection{Boundary Refinement}

The dilation threshold $t_{+}$is selected slightly larger than $t_{-}$, i.e., $t_{+}:=t_{-}+\varepsilon$ (Figure 2f) to include small irregularities in the nodule boundaries that were previously eradicated

\footnotetext{
${ }^{3}$ None was encountered during the described experiment, and our database of 700 pulmonary metastases included only a single case.
} 

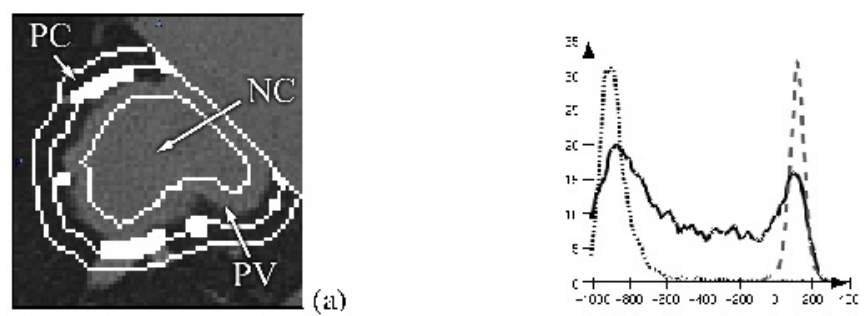

Fig. 4. (a) The white contour lines depict (from the inside to the outside) the nodule core (NC), partial volume (PV) and the parenchyma (PC) regions. The PV region is defined by the set of all voxels within $2 \mathrm{~mm}$ to the segmented nodule boundaries in either direction. The thick white areas $\left(I_{\varepsilon}\right)$ are to be excluded from the PV region, since they were determined to be vascular attachments during the boundary refinement step (Figure 2 $\mathrm{g}$ ). (b) Histograms for the parenchyma region (dotted line), the nodule core region (dashed) and the partial volume area (solid).

by the erosion procedure. Previously removed vessel and chest wall parts are added in $N_{+}:=\left\{v \in V: D(v)<t_{+}\right\}$as well. We therefore compute the intersection of the initial segmentation with the boundaries of the dilated mask, $I:=N_{0} \cap \partial N_{+}$. Dilating $I$ by $\varepsilon$ results in $I_{\varepsilon}$ (see Figure $2 \mathrm{~g}$ ), which is used to removed unwanted structures. Our final segmentation result $N_{\star}$ (Figure 2h) is then defined as

$$
N_{\star}:=N_{+} \backslash I_{\varepsilon}
$$

\subsection{Volumetry}

The segmentation procedure described above performs its boundary refinement by using the initial fixed threshold segmentation results. As shown by Ko et al. in [3], fixed threshold methods are not suitable to reproducibly assess nodule volumes, especially for smaller nodules. In contrast to the experiments performed in [3], we have to deal with vasculature and chest wall regions within the volume of interest. Thus, a variation of the PVMA method described there was developed: Based on the segmentation result $N_{\star}$, three different areas, the nodule core (NC), a parenchyma area (PC) and a partial volume region (PV) are automatically identified (Figure 4). Average attenuation values are extracted from NC and PC to allow a weighted contribution of the voxels within PV to the nodule volume. In case the region $\mathrm{NC}$ is too small to extract a reliable average, we assume a fixed mean nodule density typical for solid nodules (i. e., $50 \mathrm{HU}$ ) instead.

\section{Experiments and Results}

During the development process, the segmentation method was repeatedly applied to a database containing over 700 lung nodules from various patients and CT scanners to ensure its robustness against the variety of nodule appearances in CT data. These nodules were not used in the reproducibility tests described in the following. Reproducibility of 

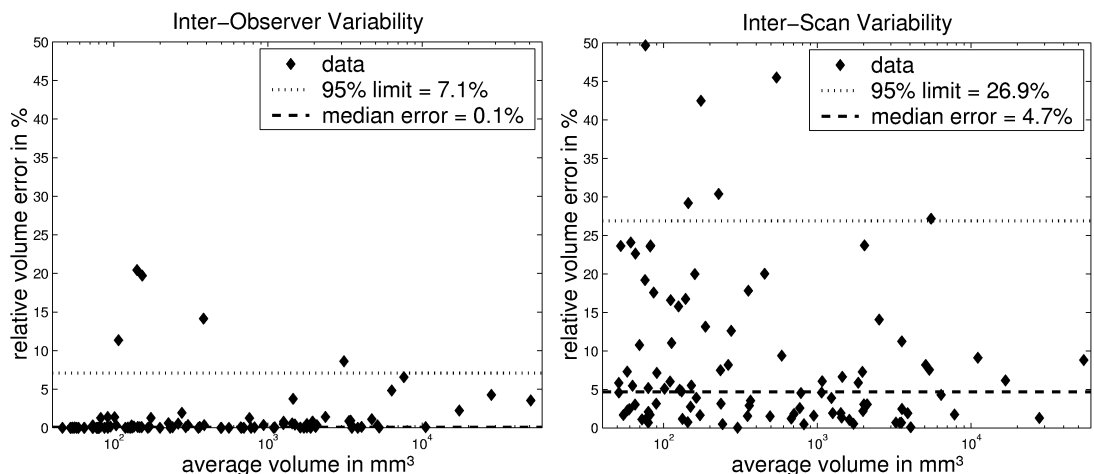

Fig. 5. Bland and Altman plots of absolute inter-observer and inter-scan variability.

volumetric results over different scans is essential with respect to tumor follow up. A study was performed using 16 CT scans (low-dose, $0.8 \mathrm{~mm}$ reconstruction increment, standard lung reconstruction kernel) from 8 patients with lung metastases acquired with a Siemens VolumeZoom CT scanner. After the first scan, the patient was asked to leave the scanner and the second low-dose scan was obtained independently from the first. The cases were processed by an expert radiologist and a radiological technician both unaware of the internals of the segmentation method. 105 lesions were identified by the radiologist with a minimum diameter of about $4.6 \mathrm{~mm}$ (the equivalent to $0.05 \mathrm{ml}$ of volume for a spherical nodule). To ensure that seed points and surrounding volumes were selected without bias, the identified locations were used only for a rough indication of the nodule positions. In addition to the principal study comparing the measured volumes in the first and second scan, inter-observer repeatability tests were performed for the 8 primary scans.

For 96 of the total of 105 nodules $(91.4 \%)$ the segmentation was classified as successful by the radiologist, the remaining 9 were excluded from the evaluation. The Bland and Altman statistic [10] was used to determine inter-observer variability as well as the agreement of volume measurement at both $\mathrm{CT}$ scans, called inter-scan variability in the following. The mean volume of the measurements was used to estimate the unknown true nodule volume. Median error and 95\% limits of agreement (meaning that an increase of the measured volume by more than the computed limit has a $95 \%$ likelihood of being real growth rather than measurement inaccuracy) were computed and included in Figure 5] The volume differences are plotted as a percentage of the mean of the two measurements in Figure 5] Computation time measurements on a $3.2 \mathrm{GHz}$ PC revealed a mean of 0.3 and a maximum of 4.1 seconds.

\section{Discussion and Future Work}

We have developed a new method for the volume assessment of lung tumors. One of the main features of the segmentation is the automated determination of an optimal erosion 
strength for the morphological opening procedure. In combination with the chest wall separation, this allows for the robust segmentation also of larger, irregularly shaped nodules attached to the lung surface and complex vasculature. An in vivo study conducted on 105 metastases demonstrated a small inter-observer variability (95\% limit of agreement: $7.1 \%$, median: $0.1 \%$ ). The $95 \%$ limit of agreement of $26.9 \%$ (median: $4.7 \%$ ) for measurements on two independent low-dose scans of the same patient shows that clinically significant volume increase can be detected reliably by the proposed method. The computation time on a PC was negligible (mean: $0.3 \mathrm{sec}$., maximum: $4.1 \mathrm{sec}$.). The evaluation of reproducibility on two independent low-dose patient CT scans is a challenging setting. Ongoing evaluation activities include phantom studies to assess variability under different reconstruction algorithms and absolute accuracy of the method. Nodules attached to concave parts of the lung surface (such as the diaphragm) appeared to be responsible for most of the larger measurement discrepancies, the improvement of their segmentation is subject of future methodological work.

By targeting the fast reproducible volumetric assessment of large pulmonary metastases, the presented method extends the applicability of $3 \mathrm{~d}$ computer assisted lung nodule volumetry to chemotherapy monitoring. By the knowledge of the authors, no applications or algorithms designed for that purpose have previously been published.

The algorithm has been integrated into an application prototype using the research and development platform MeVisLab[11]. The prototype has been installed at six German and two North American clinics for further evaluation.

Acknowledgments. The research leading to this publication has been supported by the German Federal Ministry of Education and Research under grant number 01EZ0010. It was conducted as part of the cooperation project VICORA - Virtual Institute for Computer Assisted Radiology. Special thanks go to Dr. H. Bourquain and T. Schleef for their participation in the study.

\section{References}

1. Kostis WJ; Reeves AP; Yankelevitz DF; Henschke CI: Three-dimensional segmentation and growth-rate estimation of small pulmonary nodules in helical CT images. In: IEEE Trans. on Med. Imaging, 22(10) (2003) 1259 - 1274

2. Wormanns D; Kohl G; Klotz E; et al: Volumetric measurements of pulmonary nodules at multi-row detector CT: in vivo reproducibility. In: Eur. Radiol. 14(1) (2004) 86-92.

3. Ko JP; Rusinek H; Jacobs EL; et al: Small Pulmonary Nodules: Volume Measurement at Chest CT - Phantom Study. In: Radiology, 228(3) (2003) 864-870

4. Armato SG III; Giger ML; Moran CJ; et al: Computerized detection of pulmonary nodules on CT scans. In: Radiographics, 19(5) (1999) 1303-1311

5. Brown MS; McNitt-Gray MF; Goldin JG; et al: Patient-specific models for lung nodule detection and surveillance in CT images. In: IEEE Trans. on Med. Imaging, 20 (2001) 12421250

6. Reinhardt JM; Guo J; Zhang L; et al: Integrated System for Objective Assessment of Global and Regional Lung Structure. In: Proc. of MICCAI 2001, LNCS 2208 (2001) 1384-1385

7. Kuhnigk JM; Hahn HK; Hindennach M; et al: Lung Lobe Segmentation by Anatomy-Guided 3 D Watershed Transform. In: Proc. of SPIE Medical Imaging 2003, 5032 (2003) 1482-1490 
8. Sonka M; Hlavac V; Boyle R: Image Processing, Analysis and Machine Vision; 2nd Ed; International Thomson Publishing, (1998)

9. Bruijns J: Fully-Automatic Lebelling of Aneurysm Voxels for Volume Estimation. In: Proc. of Bildverarbeitung fuer die Medizin, (2003) 51-55

10. Bland JM, Altman DG: Statistical methods for assessing agreement between two methods of clinical measurement. In: Lancet 1 (8476) (1986) 307-310

11. Hahn HK, Link F, Peitgen HO: Concepts for a Rapid Prototyping Platform in Medical Image Analysis and Visualization. In: Proc. SimVis, SCS (2003) 283-298 